\title{
YDEF Insulin Pump Course 2014
}

\author{
Dr Munachiso Nkwolo provides an overview of this \\ popular programme.
}

The popular YDEF Insulin Pump Course ran again earlier this summer. Set up by registrars in the early days of the Young Diabetologists Forum to fill the gap in the market for good quality insulin pump training, the course is delivered annually for diabetes and endocrinology trainees and new consultants.

CSII use has exploded in recent years with the 2012 United Kingdom Insulin Pump Audit revealing that 183 hospital trusts across the UK provided an insulin pump service. Despite this we in the UK are lagging behind our European and American colleagues. The audit estimated that only $6 \%$ of adults with type 1 diabetes are managed with CSII, well below the $15-20 \%$ NICE target. The authors concluded that this may well be due to a lack of training of professional staff. 1,2

So with this in mind, the YDEF has continued to support and run the 3 day Insulin Pump Course. Behind the scenes, Dr Pratik Choudhary, Senior Lecturer and Consultant at King's College Hospital, put together a faculty of internationally recognised speakers and experienced members of the pump MDT, including senior DSNs and dieticians. Day 1 began with a highly rated talk by Professor Stephanie Amiel, RD Lawrence Professor of Diabetic Medicine at King's, which emphasised the importance of truly understanding insulin physiology and optimising multiple daily injection therapy before even looking at a pump. This was followed by sessions detailing the relevant research and current guidelines. Dr Anna Brackenridge, Diabetes Consultant at Guy's and St Thomas' Hospital, shared their interpretation of NICE guidance and how their pump service has developed and progressed.

Delegates then got practical during arguably the most important part of the course; trying the pumps for themselves. Everyone was supplied with a pump from each of the main manufacturers Animas, Medtronic and Roche who kindly sponsored the meeting. Once connected trainees were taught various functions such as bolusing, basal rate settings and some interesting so- lutions for discrete storage. Pumps then stayed on for the drinks reception, dinner and overnight so delegates could carbohydrate count, bolus and reset their basal settings if, for example, they had a glass of wine or two with dinner.

Day 2 highlights included comprehensive accounts of established pump services. Dr Dennis Barnes, Clinical lead for Diabetes and Endocrinology at Maidstone and Tunbridge Wells Trust, shared the Pembury DGH experience, whilst Dr David Hopkins, Clinical Director King's College Hospital, discussed complex cases managed within the

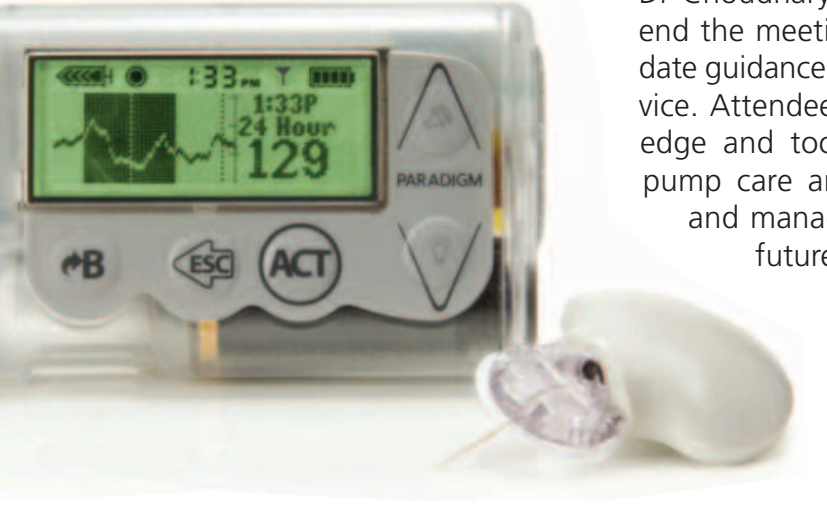

King's service. Chris Cheyette, author of Carbs \& Cals and Senior Dietician at King's College Hospital ran an interactive session on carbohydrate counting. Talks with practical advice on continuous glucose monitoring and complications such as DKA and hypoglycaemia were also delivered before the day concluded with a Pump swap shop. Delegates said goodbye to the pump they had worn for the last 24 hours and tried the different styles and settings of the other pumps on offer.

The high standard of speakers continued through to the final day. Dr Nick Oliver, Diabetes Consultant Imperial College, gave a fantastic talk on the progress of closed loop insulin delivery and the feasibility of future clinical application. Delegates were then spoilt for choice as they rotated through workshops led by specialists in their field; Pumps and pregnancy led by Dr Peter Hammond, Diabetes Service Director Harrogate Hospital; Pumps and exercise discussed

\author{
Abbreviations and acronyms \\ CSII continuous subcutaneous insulin \\ infusion \\ DKA diabetic ketoacidosis \\ DSN diabetes specialist nurse \\ NICE National Institute for Health and \\ Care Excellence \\ MDT multidisciplinary diabetes team \\ YDEF Young Diabetologists and \\ Endocrinologists' Forum
}

by $\mathrm{Dr}$ Alistair Lumb, Diabetes Consultant OCDEM and Interpreting pump downloads led by Dr Pratik Choudhary. The day closed with an 'Ask the experts' panel chaired by Dr Choudhary. It was an excellent way to end the meeting that had delivered up to date guidance, experience and practical advice. Attendees were left with the knowledge and tools to provide good quality ump care and encouraged to establish ge key pump services in the uture.

The YDEF would like to thank the faculty for their time, support and brilliant contributions. We remain grateful to Medtronic, Roche, Animas and Abbott without whom we would not be able to deliver the course and Elise Harvey for her super-efficient administration. Very special thanks to Dr Pratik Choudhary, an original founder, whose drive and enthusiasm for the course has kept it going since conception.

\section{References}

1. Grant P, Cheer K, Herring R, et al. CSIl experience among higher specialist trainees: a Young Diabetologists and Endocrinologists Forum survey. Pract Diabetes 2013;30:3325. http://dx.doi.org/10.1002/pdi.1806

2. White HD, Goenka N, Furlong NJ, et al. The UK service level audit of insulin pump therapy in adults. Diabet Med 2014;31(4):412-18. http://dx.doi.org/10.1111/dme.12325

Dr Munachiso Nkwolo

E-mail: munachiso@googlemail.com

http://dx.doi.org/10.15277/bjdvd.2014.032 Br J Diabetes Vasc Dis 2014;14:119 